\title{
16
}

\section{Evaluation of Enterprises within a Turbulent Market - Control the Dynamic Character of Turbulent Markets of the Next Millennium}

\author{
D. Markfort, C. Borgmann, N. Brehmer, T. Förster \\ Fraunhofer Institute, Factory Operation and Automation \\ Steinfeldstrasse 3, 39179 Barleben, Germany \\ markfort@iff.fhg.de
}

\begin{abstract}
The environment is turning more and more turbulent. Enterprises are not able to predict the future over a longer time and notice the drastic changes on the markets that they serve. To ensure the long time capability of survival within turbulent markets, enterprises have to generate flexible, adaptable and living organizational structures, best known as dynamic structures. To meet this demand, enterprises need instruments and methods to point out the current organizational structure and to highlight change requirements. These are prerequisites to set goals and to develop strategies to reach them.

The focal point of this paper is the description of the Structural Behavior of an organization. The structural behavior describes the ability of an enterprise to react on market changes. Consequently, the structural behavior can be divided in rigid, chaotic and dynamic. To ensure an holistic view on the entire social-technical system enterprise, the Six-Level Model was used. This model also helps to structure the characteristics of the Structural Behavior and their degrees.
\end{abstract}

\section{Keywords}

turbulent environment, structural behavior, strategic planning, dynamic production and organizational structures, change process, socio-technical system, Six-Level Model 


\section{INITIAL CONDITIONS AND PROBLEMS}

In the past, enterprises operated within a stable and less changing environment, because the vendor markets were predictable. The high efficiency of enterprises based on settled hierarchical structures and the separation of planning and working.

Since the early $80 \mathrm{ies,}$ markets getting less predictable and the complexity of enterprises and markets increases. The reaction time to respond to market changes are also increasing. These trends have the following causes:

- Fast changing markets - Market's behavior is less predictable and insecure. Long-time planning is not possible and therefore entrepreneurial mistakes are increasing.

- World wide availability of resources - Resources are available in shorter times and world wide. The relationships between vendor and buyer are valid over longer times.

- Rapid development of technology - Especially the information and communication technology is changing fast and allows world wide contacts in shorter times.

- Individualization of customer demands - Customer request individual solutions for individual problems. Enterprises have to adjust to this development.

Traditional grown structures are not able to manage these environmental demands. They are not able to follow market changes in an adequate time.

Therefore, new management concepts were created in the past 10 years. There are concepts like Total Quality Management, Lean Production, Business Reengineering, etc. Lots of enterprises realized these concepts. They minimized costs and waste and manufactured more efficiently. But they changed only ones or twice up to a higher level. These single change processes are not enough to follow the rapid environmental changes. Management plans strategically (5 to 7 years), i.e., they are planning changes within that time frame as well. But the environment will change in the future even more unpredictably. Management concepts with the goal to change enterprise structure once up to date are only partly applicable.

For surviving in a turbulent environment, today's companies need to change to dynamic structures which fulfill the requirements of adaptability, capability of development, management of complexity, robustness, self-similarity and goalorientation, and optimized usage of resources. Most important is a holistic approach by addressing changes to the company structure.

These dynamic structures are combining elements of mechanical concepts (Lean Production, TQM, etc.) with elements of organic concepts (Fractal Company, Agile Manufacturing, Learning Organization, etc.) in the right way to meet market demands. 


\section{STRUCTURAL BEHAVIOR}

How can a dynamic structure be described? This is a very complex issue that needs to be addressed from several points of view. We are also convinced, that different organizational structures are able to fulfill the mentioned demands.

This aware, the question what dynamic structures should fulfill raises. Answering this question, the scientist has a basis to compare different enterprises and their structures. One does not has to compare the organizational elements and their relationships or the amount of interfaces an order has to pass. The investigation is not only focused on process and the hierarchical structure of an enterprise.

The following abilities of dynamic structures were identified (Warnecke, 1993). Dynamic structures are:

- Able to Reconfigure - they are self-organizing, self-optimizing, adaptable and capable to develop

- Self-Similar - they are oriented towards overall objectives and similar to each other in goal orientation.

- Stable - they are successful and viable in the long run.

- Capable to Manage Complexity - they are designed from the holistic view of the company and along the processes. Organizational units are part of these dynamic structures, which are able to manage complexity through breaking down the global tasks in smaller parts that concern the unit.

Considering that, structural behavior shows the ability of the organization to follow market changes. This is independent from the actual organizational structure.

\subsection{Six-Level Model}

The description of structural behavior bases on the Six-Level Model (Kühnle, 1994). Fig. 1 shows the Model and its six horizontal levels.

This level model ensures the integral view on the enterprise. It decreases the complexity of the organization, because it subdivides the complex social-technical system enterprise into six horizontal levels. The levels culture, strategy, socialinformal level, financial and information level as well as process and layout can be searched separately but only the integral view on all levels ensures an integral analysis of the entire organization.

The level culture describes the organization as social system as well as the value and thinking pattern within the organization. Thereby, the organization is seen as culture, as partnership with defined value and orientation patterns.

The level strategy describes the ability of enterprises to serve the market with all available resources. The enterprise has to maximize customer benefits. This level describes the efficiency of organizations.

The focus of the social-informal level are the employees. It describes the relationship of all employees within the organization. The culture of the social-technical system enterprise builds the frame of this level. 


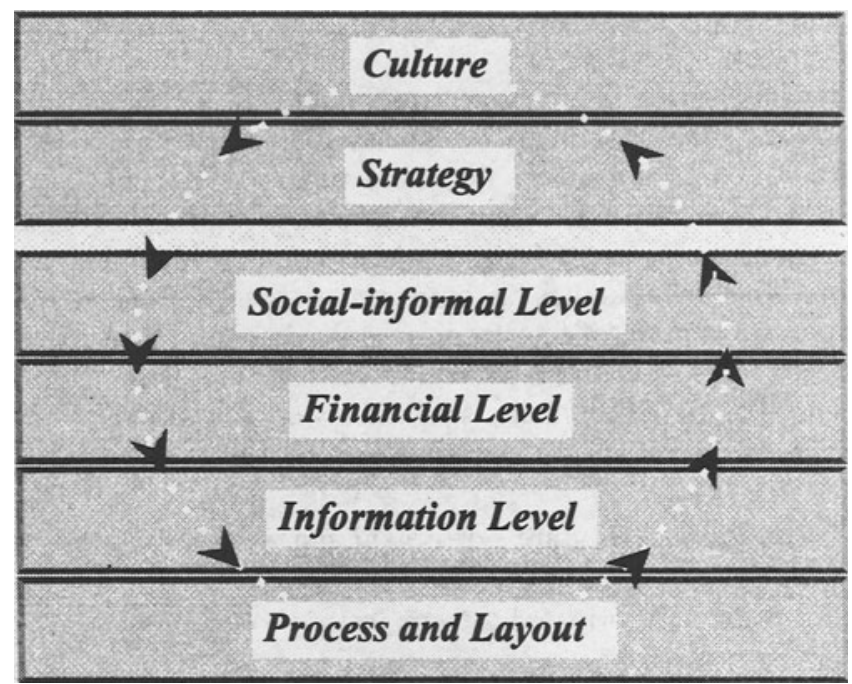

Figure 1 Six-Level Model.

The financial level analyses the economic efficiency. Focus of that level are financial processes and their individual adaptations to different organizational forms.

All technical processes and material flows within the value chain are analyzed in the level process and layout. Therefore, it is also called technical level.

To sum it up, one can say that the levels culture and strategy are building the frame for the activities within the other 4 process-levels. The culture defines the organization as social system and with the help of strategies, enterprise is able to define action patterns to ensure the efficacy of resource utilization. Therefore, this model is also called Two+Four-Level Model.

\subsection{Characteristics of Structural Behavior of an Enterprise within Turbulent Markets and their Degrees}

This Six-Level Model helps to structure the characteristics of the structural behavior and their degrees. As the results of a practical and theoretical multicompany study (Hartmann, 1997) three different degrees of structural behavior have been identified:

- rigid,

- chaotic, and

- dynamic.

The following description of these degrees of structural behavior highlights some selected characteristics of the cultural, strategic and social-informal level. 


\section{Rigid Structural Behavior}

Rigid structural behavior occurs mainly in traditional grown Enterprises. Main characteristics are the over-organized and strange hierarchical organizational structure. Management sets goals and tasks without any employee integration. The way to achieve them is dictated by management. Another characteristic of the cultural level is the degree of informal social systems. There are strongly developed informal systems within organizations with rigid structural behavior. This informal structure is not allowed but plays an important role within all organizational processes.

An important characteristic of rigid structures is the decreasing capability to react and respond to changing environmental constraints. Strategies and goals to achieve are also dictated. Because of the turbulent environment, the capability of management to consider and decide about strategic tasks decreases constantly.

A distinctive sign of rigid structural behavior on the social-informal level is e.g. the separation of competence and responsibility, known as principle of congruence (Warnecke, 1995). To master tasks, an customer order has to overcome hierarchical, horizontal and vertical obstacles and barriers.

One symptom of rigid structures are the high amount of specialists. The causes can be seen in qualifications arranged by management. Qualifications are tasksoriented, without strategic long-sightedness. There are less or no participation of employees.

\section{Chaotic Structural Behavior}

The counterpart of rigid is the chaotic structural behavior. Young enterprises or rigid factories within a fast changing environment are tending to chaos, that means instable structures.

Chaotic structural behavior is characterized through a low organizational degree that causes an uncoordinated performance of management and no or less strategic work. Management is not able to control the increasing environmental changes. Because of a weak leadership, there are no fixed organizational rules or guidelines. Single person starting activities uncoordinated and without a holistic view on the whole enterprise. The mainly small and autonomous units within young organizations are causing lots of frictional losses, redundant work and therefore they waste time.

Management also order adaptations and changes without operational and strategic necessity. They do not have enough information in the right quality to analyze the environment. Because of that, their behavior is reflexive and the enterprise tends to under- resp. overreactions.

The enterprise does not have a fixed visior or goals and their strategies are fuzzy and not clearly defined. Therefore, they are changing faster as the environment requires.

On the social-informal level, enterprise is characterized through a fuzzy assignment of tasks. Management does not dedicate the business to the employees or to a proper department. This destroys systematically the ability of employees to solve problems independently, because nobody feels responsible for the tasks to do. Every employee is waiting for the specification of the tasks or further instructions. 
Therefore, the fuzzy assignment also causes a decreasing competence and staff motivation.

However, only the comprehensive employee participation guarantees the survival of the enterprise. Management does not have a decisive influence on employee qualifications or the topics of them. The employees decide about and organize their own qualification. Results are past-oriented qualified employees.

\section{Dynamic Structural Behavior}

Creating dynamic structures management has to speed up rigid structures or has to slow down chaotic structures (refer fig. 2).

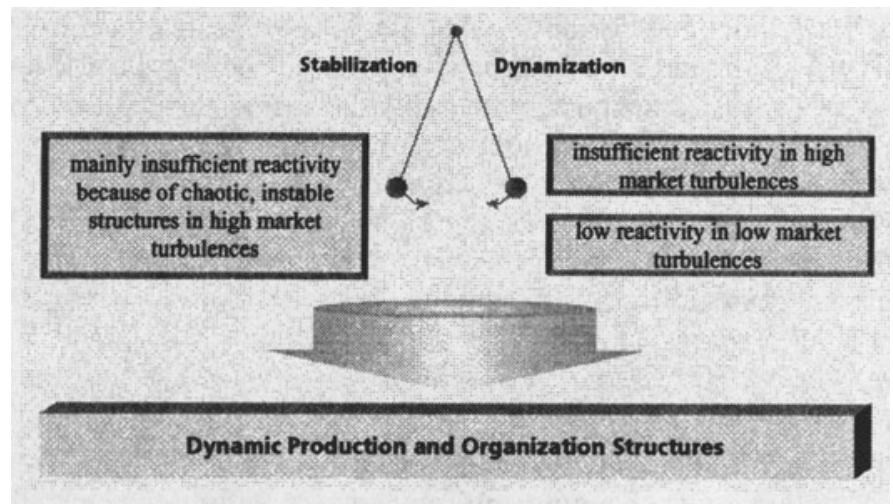

Figure 2 Creating Dynamic Structures.

Main characteristic of dynamic structural behavior on the cultural level is the arrangement of goals and tasks between management and staff. The employees participate in the way how to reach the agreed goals. The team has enough decision competencies to initiate and complete changes. The changes to do are transparent for everyone and the staff is able to influence them. Management and employees are aware of the changing environment and the need to change themselves. They are "living with the change" actively.

Organizational restrictions and rules are broad, so the employees are having enough space to act independently. This guaranties fast reactions on market demands. The employees do not have to consider high bureaucratically rules and processes that take too much time to overcome. This is one aspect that shows, that dynamic organization are operating close to the market.

The management behavior is characterized by a clear goal orientation. Strategies, goals and the vision are defined under the consideration of internal and external circumstances. The organizational vision, goals, strategies will be permanent adapted to the changing, turbulent environment. The decision making process on all hierarchical levels bases on broadly communicated information within the organization. 
The dynamic structural behavior also bases on self-organization and -optimization and self-similarity of organizational units and therefore of the whole enterprise too. These are basic elements of living, flexible and adaptable - best known as reconfigurable - structures to satisfy customer demands.

On the social-informal level, dynamic behavior bases on the congruency of task, competence and responsibility within an autonomous, decentralized organizational unit. These units are able to react fast, They are acting close together with the customer, so they also know their demands and requests. This is the basis to manufacture individual solutions for customer in a high quality and shorter times.

Management and employees arrange the qualifications. There are not only taskoriented qualification, but also methodical and social qualifications as well as the training of thinking in contexts and over the entire value chain. Continuos learning is self-evident for all members of the organization.

The above mentioned agreements in qualification, goal setting, etc. show the high level of employee participation. That is also one of the most important instruments to use the existing high potentials of the employees and to develop and extend these potentials.

\section{RESULTS}

This paper highlights some, selected characteristics of structural behavior and there degrees on the levels culture, strategy and on the social-informal level as well. The characteristics of structural behavior and their degrees are also defined on the other three levels of the Six-Level Model. Therefore, there is an complete instrument to evaluate organizational structures.

Main result is the development of a management instrument, so management is able to analyze the current structural behavior of their own organization. The analysis points not only the structural behavior out, it also highlights the requirements to change. The goal must be the implementation of dynamic structural behavior on all six levels.

Enterprises acting within turbulent markets have to ensure dynamic structural behavior. This guarantees a high level of adaptability and flexibility. Because of these characteristics, enterprises are getting more robust and increase their own competitiveness.

With the implementation of dynamic structural behavior, enterprises are only able to react on the market. Being able to act on the market, management has to implement dynamic structures. Prerequisite is, beside dynamic structural behavior, the ability to act chance driven. Only the combination of dynamic structural behavior and the ability to act chance driven ensures long-term success.

Like it is mentioned above, the generated management instrument is not only worked out theoretically. This instrument was used in ten several German enterprises. Management was able to identify the current structural behavior of their organizations. The practical usage underlines the fast and simple applicability for management. It was seen that the instrument is easy to handle and good understandable. 


\section{Next Steps}

For further research activities it is important to work completely out the change driver as the second characteristic to describe organization within a turbulent environment. The combination of these two basic characteristics of enterprises within turbulent markets opens a positioning matrix (refer fig. 3).

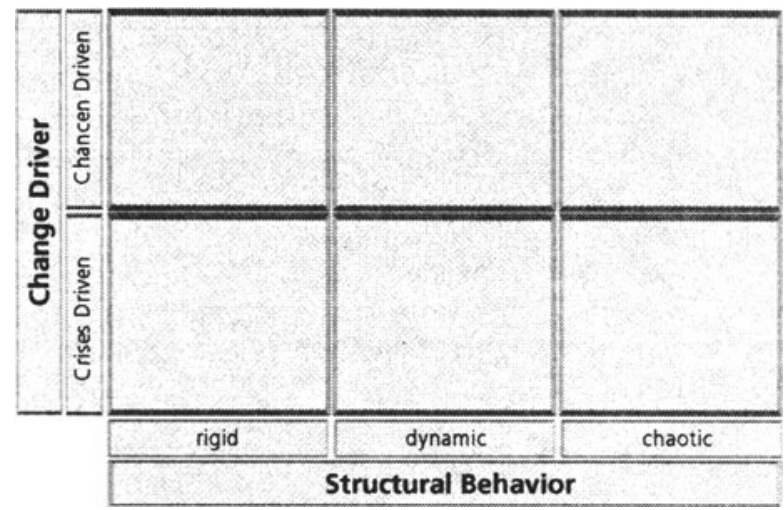

Figure 3 The Positioning Matrix.

Creating this matrix, organizations are able to position themselves within the turbulent environment. Management has an instrument to visualize the current and the goal position. Checking the current individual structural behavior and the change driver of an organization, management is able to derive requirements to change to a dynamic organization.

Considering the possible six fields within that matrix, one can create guidelines to reach the dynamic organization depending on the individual starting position.

\section{REFERENCES}

H.-J. Warnecke. (1995). Aufbruch zum fraktalen Unternehmen. Springer, Berlin, Heidelberg, New York

Kühnle, H. (1994). Die Fraktale Fabrik - Neue Organisationsformen, in: Menschen, Maschinen, Märkte (W. Kunerth). Springer, Berlin a.o.

Hartmann, M. (1996). DYNAPRO - Erfolgreich produzieren in turbulenten Märkten. Anforderungen und industrielle Lösungsansätze. LOGIS, Stuttgart

Hartmann, M. (1997). DYNAPRO - Erfolgreich produzieren in turbulenten Märkten. Methoden für dynamisches Management. LOGIS, Stuttgart 
Warnecke, H.J. (1993). The Fractal Company. A Revolution in Corporate Culture. Springer, Berlin, Heidelberg, New York

Förster, T.; Schmelzer, S.F. (1996): A New Paradigm: The Fractal View of the Company, in: NGMS International Conference, Irvine, USA, February 7, 1996 